\title{
Calibration of the Borated Ion Chamber at NIST Reactor Thermal Column
}

\author{
Z. Wang and N. E. Hertel \\ G. W. Woodruff School of Mechanical Engineering \\ Georgia Institute of Technology, \\ Atlanta, Georgia, USA, 30332-0405 \\ A. Lennox \\ Fermi National Accelerator Laboratory \\ Fermilab Mail Stop301, Kirk and Wilson Streets, \\ Batavia, Illinois, USA 60510
}

\begin{abstract}
In Boron Neutron Capture Therapy (BNCT) and Boron Neutron Capture Enhanced Fast Neutron therapy (BNCEFNT), the absorbed dose of tissue due to the boron neutron capture reaction is difficult to measure directly. This dose can be computed from the measured thermal neutron fluence rate and the ${ }^{10} \mathrm{~B}$ concentration at the site of interest. A borated tissue-equivalent (TE) ion chamber can be used to directly measure the boron dose in a phantom under irradiation by a neutron beam. Fermilab has two Exradin 0.5$\mathrm{cm}^{3}$ Spokas thimble TE ion chambers, one loaded with boron, available for such measurements. At the Fermilab Neutron Therapy Facility, these ion chambers are generally used with air as the filling gas. Since alpha particles and lithium ions from the ${ }^{10} \mathrm{~B}(\mathrm{n},)^{7} \mathrm{Li}$ reactions have very short ranges in air, the Bragg-Gray principle may not be satisfied for the borated TE ion chamber.

The two TE ion chambers were calibrated in the thermal column of the National Institute of Standards and Technology (NIST) research reactor. The borated TE ion chamber is loaded with $1000 \mathrm{ppm}$ of natural boron $\left(184 \mathrm{ppm}\right.$ of $\left.{ }^{10} \mathrm{~B}\right)$. The NIST thermal column has a cadmium ratio of greater than 400 as determined by gold activation. The thermal neutron fluence rate during the calibration was determined using a NIST fission chamber to an accuracy of $5.1 \%$. The chambers were calibrated at two different thermal neutron fluence rates: $5.11 \times 10^{6}$ and $4.46 \times 10^{7} \mathrm{n} \mathrm{cm}^{-2} \mathrm{~s}^{-1}$. The non-borated ion chamber reading was used to subtract collected charge not due to boron neutron capture reactions. An optically thick lithium slab was used to attenuate the thermal neutrons from the neutron beam port so the responses of the chambers could be corrected for fast neutrons and gamma rays in the beam. The calibration factor of the borated ion chamber was determined to be 1.83 $\mathrm{x} 10^{9} \pm 5.5 \% \mathrm{n} / \mathrm{cm}^{2}$ per $\mathrm{nC}$ at standard temperature and pressure (STP) condition.
\end{abstract}




\section{Introduction}

In Boron Neutron Capture Therapy (BNCT) and Boron Neutron Capture Enhanced Fast Neutron therapy (BNCEFNT), the absorbed dose of tissue due to the boron neutron capture reaction is difficult to measure directly. This dose often is computed from the measured thermal neutron fluence rate and the ${ }^{10} \mathrm{~B}$ concentration at the site of interest. $\mathrm{A}$ borated tissue-equivalent (TE) ion chamber can be used to directly measure the boron dose in a phantom under irradiation by a neutron beam. Fermilab has two Spokas TE ion chambers, one loaded with boron and the other without boron, available for such measurements. At the Fermilab Neutron Therapy Facility, these ion chambers are generally used with air as the filling gas. Since alpha particles and lithium ions from the ${ }^{10} \mathrm{~B}(\mathrm{n},)^{7} \mathrm{Li}$ reactions have very short ranges in air, the Bragg-Gray principle may not be satisfied for the borated TE ion chamber. So this chamber was calibrated in a thermal neutron beam.

The National Institute of Standards and Technology (NIST) Center for Neutron Research (NCNR) in Gaithersburg, MD has a thermal column that provides a uniform beam of thermal neutrons for dosimetry and neutron standards work. The cadmium ratio based on gold activation in the thermal neutron beam is greater than 400 . The calibration of the boron-loaded TE ion chamber was performed at the center of the beam. The thermal neutron fluence rate was determined by a dual fission ion chamber (Grundl et al., 1975) provided by NIST. The thermal neutron component of the beam can be substantially attenuated by placing an optically thick lithium slab at the opening of the port.

The two Exradin ion chambers used in Fermilab Fast Neutron Therapy facility are Spokas thimble type with a collecting volume of $0.5-\mathrm{cm}^{3}$ and are made of A150 tissueequivalent plastic. One (SSN: 446B) of the chambers, hereafter referred to as the borated chamber, is loaded with $1000 \mathrm{ppm}$ natural boron $\left(184 \mathrm{ppm}\right.$ of $\left.{ }^{10} \mathrm{~B}\right)$ in the TE material. The other chamber (SSN: 445) will be referred to as non-borated. Both of the chambers worked at +400 Volts. A Keithley 617 electrometer was used to measure the charge of the chambers.

\section{Determination of the neutron fluence rate using the fission chamber}

The conversion factor ( $\mathrm{n} / \mathrm{cm}^{2}$ per count) of the dual fission ion chamber is calculated using the number of ${ }^{235} \mathrm{U}$ nuclei, the thermal neutron fission cross section and a series of corrections. The fission cross section of ${ }^{235} U$ for thermal neutrons can be calculated by

$$
\sigma_{t h}=\frac{\sqrt{\pi}}{2} \cdot g(T) \cdot \sqrt{\frac{T_{0}}{T}} \cdot \sigma_{f}\left(E_{0}\right)
$$

where $f\left(E_{0}\right)$ is the microscopic fission cross section for neutrons of energy $E_{0}$, typically $0.0253 \mathrm{eV}$ or $2200 \mathrm{~m} / \mathrm{s}, f(0.0253 \mathrm{eV})=584 \mathrm{barn} ; T_{0}$ is the temperature of the tabulated cross section, $294.61 \mathrm{~K} ; \mathrm{T}$ is the temperature of the moderator for the measurement, 303 $\mathrm{K} ; g(T)$ is the empirical correction factor of the departure from $1 / \mathrm{v}$ behavior of ${ }^{235} \mathrm{U}$. At 
$303 \mathrm{~K}, g(T)$ is 0.974 . Substituting the values of the parameters in equation (1), $\sigma_{t h}=496 \mathrm{~b}$ is obtained.

The upper chamber of the dual fission chamber has $378.5 \pm 1 \%$ g of ${ }^{235} \mathrm{U}$ uniformly deposited over an area of $1.2668 \mathrm{~cm}^{2}$, corresponding to $9.655 \times 10^{17}{ }^{235} \mathrm{U}$ nuclei per $\mathrm{cm}^{2}$. The bottom chamber was not used in the measurements. The detection of the fission fragments is essentially $100 \%$ and the conversion factor (CF) of the fission chamber counts to thermal neutron fluence rate is

$$
C F=\frac{1}{\sigma_{t h} \cdot N}=2079 \mathrm{~cm}^{-2} / \text { count }
$$

The reading of the counter is the integration above the low level discriminator which is above zero, and the true counts need to be extrapolated to zero. The extrapolation-to-zero correction is 1.0368 , self-absorption 1.0206 , inscatter from substrate $1 / 1.034$, in-scatter from chamber $1 / 1.023$, out-scatter by chamber bottom and anode 1.009 . The total correction is $(1.0368)(1.0206)\left(\frac{1}{1.034}\right)\left(\frac{1}{1.023}\right)(1.009)=1.009$, so the corrected count-tofluence conversion factor for the fission chamber is $(2079)(1.009)=2099 \mathrm{~cm}^{-2} /$ count.

A correction for the dead time of the electronics should also be made. The width of each pulse is 2_s and the fraction of count loss due to dead time is (2_s/pulse)(\# pulses/s). The count rate of the fission chamber at low neutron fluence rate was 2417 counts per second and at high neutron fluence rate 20,426 counts per second, leading to a dead time loss of 0.005 and 0.0407 , respectively. The conversion factor of the fission chamber corrected for dead time, $C F_{f}$, are $2109.5 \pm 5 \% \mathrm{n} / \mathrm{cm}^{2}$ per count at the lower neutron fluence rate and $2184.4 \pm 5 \% \mathrm{n} / \mathrm{cm}^{2}$ per count at the higher neutron fluence rate. The thermal neutron fluence rate, $\phi_{t h}$, is determined by

$$
\phi_{i h}=C \cdot C F_{f}
$$

where $\mathrm{C}$ is the count rate (cps) of the fission chamber for thermal neutrons.

\section{Calibration coefficient of the borated TE ion chamber}

The total charge collected by the borated TE ion chamber in the thermal neutron beam, $Q^{B}$, results from particles and ions emitted in ${ }^{10} \mathrm{~B}(\mathrm{n},)^{7} \mathrm{Li}$ and ${ }^{14} \mathrm{~N}(\mathrm{n}, \mathrm{p}){ }^{14} \mathrm{C}$ reactions induced by thermal neutrons (A150 TE plastic contains 3.5\% of nitrogen by weight), from recoil nuclei and ions induced by fast neutrons and from secondary electrons produced by photons. At the NIST thermal column, thermal neutrons can be fully attenuated by placing an optically thick lithium slab placing in front of the neutron beam port. With this attenuator in place, the charge collected in the chamber due to fast neutrons and photons is recorded as $Q_{F G}^{B}$. The charge due to thermal neutrons, $Q^{B}$ th, is the difference of the above two quantities.

$$
Q_{t h}^{B}=Q_{T}^{B}-Q_{F G}^{B}
$$


The charges collected by the non-borated ion chamber due to thermal neutrons can be obtained by the same way except that $Q^{N-B}$ does not have the ${ }^{10} \mathrm{~B}(\mathrm{n},]^{7} \mathrm{Li}$ reaction contribution.

$$
Q_{i h}^{N-B}=Q_{T}^{N-B}-Q_{F G}^{N-B}
$$

The charge due to the ${ }^{10} \mathrm{~B}(\mathrm{n},)^{7} \mathrm{Li}$ reaction in the borated ion chamber is then

$$
Q_{B}=Q_{t h}^{B}-Q_{t h}^{N-B}\left(\frac{N_{C}^{N-B}}{N_{C}^{B}}\right)
$$

where $\left(\frac{N_{C}^{N-B}}{N_{C}^{B}}\right)$ is the ratio of the calibration coefficients of the non-borated and borated ion chamber in a NIST traceable ${ }^{60} \mathrm{Co}$ field. This ratio previously was determined to be 0.951 (Sweezy, 2002).

The conversion coefficient of the borated ion chamber charge to thermal fluence is thus

$$
N_{t h}=\frac{\phi_{t h}}{Q_{B}}=\frac{C \cdot C F_{f}}{Q_{t h}^{B}-(0.951) Q_{t h}^{N-B}}
$$

Or

$$
N_{t h}=\frac{\phi_{t h}}{Q_{B}}=\frac{C \cdot C F_{f}}{Q_{T}^{B}-(0.951) Q_{T}^{N-B}}
$$

The subscript $T$ in equation (8) stands for the total, including charges induced by thermal neutrons, fast neutrons and gamma rays. Charges in equation (7) are due to thermal neutrons, where fast neutrons and gamma rays induced charges are subtracted by placing the optically thick lithium plate at the beam port. Since the two ion chambers are identical and the results obtained from equations (7) and (8) should be in agreement. All readings from the ion chambers are corrected to standard temperature and pressure of $273.15 \mathrm{~K}$ and $100 \mathrm{kPa}$ (STP) by multiplying by the following correction factor,

$$
K_{T P}=\frac{273.15+T_{M}\left({ }^{\circ} \mathrm{C}\right)}{273.15} \cdot \frac{100 \mathrm{kPa}}{P_{M}(\mathrm{kPa})}
$$

where $T_{M}$ is the measurement temperature in ${ }^{\circ} \mathrm{C}$ and $P_{M}$ is the measurement pressure in $\mathrm{kPa}$.

\section{Calibration procedure}

The ion chambers, the electrometer, and the high voltage unit were placed near the NIST thermal column beam. Since they are filled with ambient air, the ion chambers have the same temperature and pressure as the experimental environment. The temperature and the pressure in the room were recorded. The fission chamber was placed at the center of the neutron beam with its front surface perpendicular to the neutron beam. The position of the chamber stem was marked to ensure that the placement of the fission chamber was 
repeatable. The fission chamber was replaced with the ion chamber and the ion chamber was centered at the same location as the fission chamber.

The boron curtain on the thermal column beam was lifted until the thermal neutron fluence rate was around $5 \times 10^{6} \mathrm{n} / \mathrm{cm}^{2}$ based on the fission chamber readings. The readings of the fission chamber were recorded several times to reduce statistical uncertainties in the count rate. The fission chamber was then replaced with the borated ion chamber (446B). An electrometer was used in charge mode, and readings for integration times of 1 second, 10 seconds and 1 minute were taken at least six times. After the measurements the optically thick lithium plate was placed in the front of the neutron beam port to stop the thermal neutrons and the responses of the borated ion chamber to fast neutrons and gamma rays were recorded. The procedure was repeated for the non-borated chamber (445).

The boron curtain in the reactor was lifted higher to obtain a larger neutron fluence rate and the calibration procedure was repeated. When the measurements with the two TE ion chambers were finished, measurements were performed with the fission chamber at three positions along the neutron beam axis and three positions about the center line of the beam perpendicular to the neutron beam. These data were used to evaluate the uncertainties caused by positioning of the chambers.

\section{Results}

The count rates of the fission chamber for the two neutron fluence rate level were shown in Table 1. The conversion and the determined thermal neutron fluence rate are also displayed in Table 1. The calibration data of the borated ion chamber and non-borated ion chamber at low and high neutron fluence rate were shown in Table 2 and 3 , respectively. The calibration for the borated ion chamber shown in Tables 2 and 3 were corrected to the standard condition (temperature of $293.15 \mathrm{~K}$ and pressure $100 \mathrm{kPa}$ ).

The calibration coefficients calculated from equations (7) and (8) agreed within $1 \%$. Since the dose due to fast neutron predominates in the fast neutron therapy beam and there is no way to separate the two components, the calibration factor of equation 8 is more appropriate to use in proposed measurements at Fermilab. The recommended calibration factor for the borated ion chamber is the average of the two values calculated using equation (8) at the two neutron fluence rates, namely, $1.83 \times 10^{6} \pm 5.5 \% \mathrm{n} / \mathrm{cm}^{2}$ per $\mathrm{pC}$ at STP conditions.

The count rates of the fission chamber at three positions along the axis of the neutron beam port and at three positions perpendicular to the axis are shown in Table 4. The count rates of the fission chamber as a function of the positions were fit from the measurements and shown in the last row of Table 4. The position of $0 \mathrm{~cm}$ is the (original) position where the thermal neutron fluence rate was determined and ion chambers calibrated. Along the beam part axis, the position with a negative sign indicates the position is closer to the reactor. For the vertical situation, it is unlikely that the chambers were placed lower than the original position, only two positions higher than the original 
position were measured. The fit equation can be used to estimate the uncertainty due to positioning of the fission chamber and the ion chambers.

Table 1. Fission chamber count rates at two neutron fluence rate level

\begin{tabular}{|c|c|c|c|c|}
\hline neutron fluence & \multicolumn{2}{|c|}{ Low } & \multicolumn{2}{|c|}{ High } \\
\hline dead time loss & $0.5 \%$ & & $4.07 \%$ & \\
\hline$\overline{C F}$ & $2109.5 \pm 5.0^{\circ}$ & $\mathrm{m}^{2}$ per count & $2184.4 \pm 5.0 \%$ & $\mathrm{n} / \mathrm{cm}^{2}$ per count \\
\hline \multirow{5}{*}{$\mathrm{C}$ (cps) } & beam port open & Li slab at port & beam port open & Li slab at port \\
\hline & 2416 & 0.19 & 20410 & 2.9 \\
\hline & 2408 & 0.35 & 20389 & 3.2 \\
\hline & 2425 & 0.36 & 20406 & 3.1 \\
\hline & 2419 & 0.30 & 20456 & 3.0 \\
\hline Average & 2417 & 0.3 & 20426 & 3.1 \\
\hline uncertainty & $0.3 \%$ & $26.0 \%$ & $0.2 \%$ & $5.1 \%$ \\
\hline$\phi_{t h}\left(\mathrm{n} / \mathrm{cm}^{2}\right)$ & \multicolumn{2}{|c|}{$5.098 \mathrm{E}+06$} & \multicolumn{2}{|c|}{$4.461 E+07$} \\
\hline
\end{tabular}

Table 2. Ion chamber readings and calibration factor at low neutron fluence rate beam

\begin{tabular}{|c|c|c|c|c|}
\hline Ion chamber & \multicolumn{2}{|c|}{$446 \mathrm{~B}$ (Borated) (pC/s) } & \multicolumn{2}{|c|}{445 (non-borated) $(\mathrm{pC} / \mathrm{s})$} \\
\hline Current $(\mathrm{pC} / \mathrm{s})$ & $Q_{T}^{\beta}$ & $Q_{F G}^{B}$ & $Q_{T}^{N-B}$ & $Q_{F G}^{N-B}$ \\
\hline & beam port open & Li slab at port & beam port open & Li slab at port \\
\hline $1 \mathrm{~s}$ & 2.98 & & & \\
\hline $10 \mathrm{~s}$ & 3.05 & 0.078 & 0.295 & 0.093 \\
\hline $1 \mathrm{~min}$ & 3.03 & 0.073 & 0.288 & 0.091 \\
\hline Average & 3.02 & 0.075 & 0.291 & 0.092 \\
\hline uncertainty & $1.1 \%$ & $4.8 \%$ & $1.8 \%$ & $1.6 \%$ \\
\hline$\left(\mathrm{n} / \mathrm{cm}^{2}{ }^{N_{t h}}\right.$ per pC) & & $\begin{array}{ll}35 \times 10^{6} & \text { (at 27 } \\
36 \times 10^{6} & \text { (at 27 }\end{array}$ & $\begin{array}{l}(100 \mathrm{kPa})(\mathrm{Eq} . \\
(100 \mathrm{kPa})(\mathrm{Eq} .\end{array}$ & \\
\hline
\end{tabular}

Table 3 Ion chamber readings and calibration factor at high neutron fluence rate beam

\begin{tabular}{|c|c|c|c|c|}
\hline Ion chamber & \multicolumn{2}{|c|}{ 446B (Borated) } & \multicolumn{2}{|c|}{445 (non-borated) } \\
\hline Current $(\mathrm{pC} / \mathrm{s})$ & $Q_{T}^{B}$ & $Q_{F G}^{B}$ & $Q_{T}^{N-B}$ & $Q_{F G}^{N-B}$ \\
\hline & beam port open & Li slab at port & beam port open & Li slab at port \\
\hline $1 \mathrm{~s}$ & 26.44 & & & \\
\hline $10 \mathrm{~s}$ & 26.44 & 0.356 & 1.883 & 0.360 \\
\hline $1 \mathrm{~min}$ & 26.36 & 0.343 & 1.901 & 0.351 \\
\hline Average & 26.42 & 0.349 & 1.893 & 0.356 \\
\hline uncertainty & $0.2 \%$ & $2.6 \%$ & $0.6 \%$ & $1.9 \%$ \\
\hline$\left(\mathrm{n} / \mathrm{cm}^{2}{ }^{N_{t h}}\right.$ per pC) & & $\begin{array}{l}1.81 \times 10^{6} \text { (at 27 } \\
1.81 \times 10^{6} \text { (at 27 }\end{array}$ & $\begin{array}{l}\mathrm{K} 100 \mathrm{kPa})(\mathrm{Eq} \\
\mathrm{K} 100 \mathrm{kPa})(\mathrm{Eq} .\end{array}$ & \\
\hline
\end{tabular}

Table 4. Evaluation of the uncertainty due to the positioning of the fission chamber

\begin{tabular}{|c|c|c|c|}
\hline \multicolumn{2}{|c|}{ Along the beam port axis $(X)$} & \multicolumn{2}{|c|}{ perpendicular to beam $(\mathrm{Z})$} \\
\hline $\mathrm{X}($ position $\mathrm{cm})$ & $\mathrm{C}($ Count rates cps $)$ & $\mathrm{Z}$ (position $\mathrm{cm}$ ) & $\mathrm{C}$ (Count rates cps) \\
\hline-0.9525 & 2552 & 0 & 2419 \\
\hline 0 & 2419 & 0.635 & 2464 \\
\hline 0.9525 & 2368 & 1.27 & 2499 \\
\hline \multicolumn{2}{|c|}{$C=2418.9-96.9 X+45.4 X^{2}$} & \multicolumn{2}{|c|}{$C=2418.9+78.8 Z-12.1 Z^{2}$} \\
\hline
\end{tabular}




\section{Discussion}

The uncertainty of the count-to-fluence rate conversion of the fission chamber was provided by NIST to be $5.0 \%$. The uncertainty of the count rates of the fission chamber due to the positioning was estimated to be $1.0 \%$. So the overall uncertainty of the thermal neutron fluence rate was $5.1 \%$. The uncertainty in the charge collected in the borated ion chamber resulted from two sources, one from the subtraction of charge due to fast neutron and photons, about $1.3 \%$, and the other from the positioning of the ion chamber, about $1.5 \%$. So the combined uncertainty in the charge collected by the borated chamber is approximately $2.0 \%$. The combined uncertainty of the calibration factor of the borated ion chamber is then $5.5 \%$.

\section{Acknowledgements}

Dr. David Gilliam at the NIST reactor provided us with the fission chamber and related electronics for the determination of thermal neutron fluence rate at the thermal column. He also helped us to make corrections for the fission chamber conversion factor. The authors also want to thank the NIST for providing the thermal neutron beam facility.

\section{Reference}

J. E. Sweezy, Development of a Boron Neutron Capture Enhanced Fast Neutron Therapy Beam, PhD. thesis, Georgia Institute of Technology, 2002.

J. E. Sweezy, A. Lennox, and N.E. Hertel, Paired Tissue Equivalent Ionization Chambers for Boron Enhancement Fast Neutron Therapy, $10^{\text {th }}$ International Congression Neutron Capture Therapy, NCT Essen 2002, September 8-11, 2002, Essen, Germany.

J. A. Grundl, D. M. Gilliam, N.D. Dudey, and R.J. Popek, Measurement of absolute fission rates, Nuclear Technology, Vol 25, pp.273-257, 1975 